\title{
Classifying spaces of twisted loop groups
}

\author{
THOMAS J BAIRD
}

\begin{abstract}
We study the classifying space of a twisted loop group $L_{\sigma} G$, where $G$ is a compact Lie group and $\sigma$ is an automorphism of $G$ of finite order modulo inner automorphisms. Equivalently, we study the $\sigma$-twisted adjoint action of $G$ on itself. We derive a formula for the cohomology ring $H^{*}\left(B L_{\sigma} G\right)$ and explicitly carry out the calculation for all automorphisms of simple Lie groups. More generally, we derive a formula for the equivariant cohomology of compact Lie group actions with constant rank stabilizers.
\end{abstract}

22E67; 57S15

\section{Introduction}

Let $G$ be a compact connected Lie group and let $\sigma \in \operatorname{Aut}(G)$ be an automorphism of $G$. The twisted loop group $L_{\sigma} G$ is the topological group $L_{\sigma} G$ of continuous paths $\gamma: I \rightarrow G$ satisfying $\gamma(1)=\sigma(\gamma(0))$, with point wise multiplication and compact-open topology. In the special case that $\sigma$ is the identity automorphism, $L_{\sigma} G$ is the usual (continuous) loop group $L G .^{1}$ The main result of this paper is a formula for the cohomology ring of the classifying space $H^{*}\left(B L_{\sigma} G\right)$.

The isomorphism type of $L_{\sigma} G$ depends only on the outer automorphism $[\sigma] \in \operatorname{Out}(G)=$ $\operatorname{Aut}(G) / \operatorname{Inn}(G)$ represented by $\sigma$; see Section 4. If $G$ is semisimple, then the outer automorphism group $\operatorname{Out}(G)$ is naturally isomorphic to the automorphism group of the Dynkin diagram of $G$, so $\operatorname{Out}(G)$ is finite.

Theorem 1.1 Let $G$ be a semisimple compact connected Lie group with Weyl group $W$ and let $\sigma \in \operatorname{Aut}(G)$ be an automorphism with corresponding outer automorphism $[\sigma] \in \operatorname{Out}(G)$. Let $G^{\sigma}$ denote the subgroup of elements fixed by $\sigma$, with identity component $G_{0}^{\sigma}$. Then the inclusion of $G_{0}^{\sigma} \subseteq G$ induces an injection in cohomology

$$
H^{*}\left(B L_{\sigma} G ; F\right) \hookrightarrow H^{*}\left(B L G_{0}^{\sigma} ; F\right)
$$

${ }^{1}$ We work with continuous loops throughout, but by work of Palais [8, Theorem 13.14] the homotopy type of $B L_{\sigma} G$ is unchanged if we work for $C^{k}$ loops for $k \geq 0$ or $L_{p}^{k}$ loops for $k>1 / p$ 
for coefficient fields $F$ of characteristic coprime to the order of $W$, the order of $[\sigma]$, and to the number of path components of $G^{\sigma}$. The image of the injection is the ring of invariants

$$
H^{*}\left(B L_{\sigma} G ; F\right) \cong H^{*}\left(B L G_{0}^{\sigma} ; F\right)^{W_{\sigma}}
$$

under an action by a subgroup $W_{\sigma} \subseteq W$. Specifically, $W_{\sigma}=N_{G}\left(T^{\sigma}\right) / T$ is the quotient of the normalizer of a maximal torus $T^{\sigma} \subseteq G_{0}^{\sigma}$ by a maximal torus $T \subseteq G$.

In many cases (see Section 6), $W_{\sigma}$ acts via outer automorphisms of $G_{0}^{\sigma}$, which are well understand. Classifying spaces of untwisted loop groups are also well understood (see Proposition 7.2) so Theorem 1.1 enables explicit calculation of $H^{*}\left(B L_{\sigma} G ; F\right)$ whenever the hypotheses hold. We carry out this calculation for all automorphisms of compact connected simple Lie groups in Section 7.

More generally, we derive a formula in Proposition 4.1 for $H^{*}\left(B L_{\sigma} G ; F\right)$ if $G$ is compact connected and $\sigma$ is conjugate to an automorphism of finite order. The proof uses the following model of $B L_{\sigma} G$. Denote by $G_{\mathrm{Ad}_{\sigma}}$ the left $G$-space whose underlying space is the group manifold $G$ and with twisted adjoint action

$$
\operatorname{Ad}_{\sigma}: G \times G_{\mathrm{Ad}_{\sigma}} \rightarrow G_{\mathrm{Ad}_{\sigma}}, \quad \operatorname{Ad}_{\sigma}(g)(x)=g x \sigma\left(g^{-1}\right) .
$$

The classifying space $B L_{\sigma} G$ is homotopy equivalent to the homotopy quotient $E G \times_{G}$ $G_{\mathrm{Ad}_{\sigma}}$; see Lemma 4.2.

If $\sigma$ has order $n$, then form the compact semi-direct product $\mathbb{Z}_{n} \ltimes G$ by the rule $(a, g) \cdot(b, h)=\left(a+b, \sigma^{-b}(g) h\right)$. The $G$-space $G_{\operatorname{Ad}_{\sigma}}$ is identified with the standard adjoint action of $G \cong\{0\} \times G$ on the path component $\{1\} \times G$. By a result of de Siebenthal [11, last theorem of Chapter II], the stabilizers $G_{p}$ of this action all have the same rank; this permits us to apply the following result which may be of more general interest.

Theorem 1.2 Let $G$ be a compact connected Lie group and $X$ a compact connected Hausdorff $G$-space with constant rank stabilizers. Choose any $p \in X$ and let $T_{p} \subseteq G$ be a maximal torus in the stabilizer $G_{p}$ of $p$. Then the inclusions $N_{G}\left(T_{p}\right) \subseteq G$ and $X^{T_{p}} \subseteq X$ induce an isomorphism in equivariant cohomology

$$
H_{G}^{*}(X) \cong H_{N_{G}\left(T_{p}\right)}^{*}\left(X^{T_{p}}\right)
$$

for coefficient fields of characteristic coprime to order of the Weyl group of $G$.

The proof of Theorem 1.2 is a straightforward generalization of the special case proven in Baird [2, Theorem 3.3] where the stabilizers $G_{p}$ were assumed to have rank equal to that of $G$. 
Twisted loop groups have been studied in relation to the representation theory of affine Lie algebras by Pressley and Segal [9], Mohrdieck and Wendt [7] and Wendt [13], and in relation to Wess-Zumino-Witten theory by Stanciu [12]. We introduced a special class of twisted loop groups - called real loop groups [3] — in the course of calculating $\mathbb{Z}_{2}$-Betti numbers of moduli spaces of real vector bundles over a real curve. The results of the current paper will be applied in future work to study the cohomology of these moduli spaces in odd characteristic.

Notation Given a topological group $G$ and $G$-space $X$, we denote by $X_{h G}$ or $E G \times_{G} X$ the Borel construction homotopy quotient.

Acknowledgements I would like to thank Misha Kotchetov for helpful advice about triality and the referee for pointing towards a gap in a proof appearing in an earlier draft. This research was supported by an NSERC Discovery Grant.

\section{Cohomological principal bundles}

We recall some background material from [2]. Let $f: X \rightarrow Y$ be a continuous map between topological spaces $X$ and $Y$, and let $\Gamma$ be a topological group acting freely on $X$, such that $X \rightarrow X / \Gamma$ is a principal bundle.

Definition 1 We say $(f: X \rightarrow Y, \Gamma)$ is a cohomological principal bundle for the cohomology theory $H^{*}$ if:

(i) $f$ is a closed surjection.

(ii) $f$ descends through the quotient to a map $h$ :

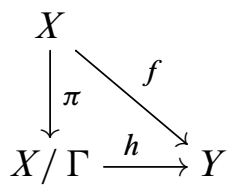

(iii) $H^{*}\left(h^{-1}(y)\right) \cong H^{*}(\mathrm{pt})$ for all $y \in Y$.

Let $H^{*}(X ; F)$ denote sheaf cohomology of the constant sheaf $F_{X}$, where $F$ is a field. The following is a simplification of [2, Corollary 2.4].

Proposition 2.1 Let $\Gamma$ be a compact Lie group and let $(f: X \rightarrow Y, \Gamma)$ be a cohomological principal bundle for $H^{*}(\cdot, F)$, where $X$ is a paracompact Hausdorff space. Then $H^{*}(Y ; F) \cong H^{*}(X / \Gamma ; F)$. 
We also make repeated use of the following.

Theorem 2.2 [2, Theorem 2.2] Let $X$ be a topological space, let $\Gamma$ be a finite group acting on $X$ and let $\pi: X \rightarrow X / \Gamma$ denote the quotient map onto the orbit space $X / \Gamma$. If $F$ is a field satisfying $\operatorname{gcd}(\operatorname{char}(F), \# \Gamma)=1$, then

$$
\pi^{*}: H(X / \Gamma ; F) \rightarrow H(X ; F)^{\Gamma}
$$

is an isomorphism, where $H(X ; F)^{\Gamma}$ denotes the ring of $\Gamma$ invariants.

A particular example of Theorem 2.2 is that $B \Gamma$ is acyclic for coefficient field $F$ coprime to the order of $\Gamma$, because $H^{*}(B \Gamma ; F)=H^{*}(E \Gamma / \Gamma ; F)=H^{*}(E \Gamma ; F)^{\Gamma}$ and $E \Gamma$ is acyclic because it is contractible.

\section{Cohomology of $G$-spaces with constant rank stabilizers}

Let $G$ be a compact Lie group and $X$ a left $G$-space which is compact connected and Hausdorff. Denote by $G_{X}$ the stabilizer of a point $x \in X$ and by $G_{X}^{0}$ the identity component of $G_{X}$. For a given point $p \in X$, let $T_{p}$ denote a maximal torus in the identity component $G_{p}^{0}$ of $G_{p}$. Define an equivariant map

$$
\phi: G \times X^{T_{p}} \rightarrow X, \quad \phi((g, x))=g \cdot x,
$$

where $G$ acts on $G \times X^{T_{p}}$ by $g \cdot(h, x)=(g h, x)$. The normalizer $N\left(T_{p}\right)=N_{G}\left(T_{p}\right)$, acts on $G \times X^{T_{p}}$ from the right by

$$
(g, x) \cdot n=\left(g n, n^{-1} \cdot x\right),
$$

leaving $\phi$ invariant and commuting with the $G$ action.

Proposition 3.1 Under the hypotheses of Theorem 1.2, the pair ( $\phi: G \times X^{T_{p}} \rightarrow$ $\left.X, N\left(T_{p}\right)\right)$ is a cohomological principal bundle.

We begin with a few of lemmas.

Lemma 3.2 Under the hypotheses of Theorem 1.2, given any two points $x, y \in X$, the maximal tori $T_{x} \subseteq G_{x}^{0}$ and $T_{y} \subseteq G_{y}^{0}$ are conjugate in $G$.

Proof Given $p \in X$, the set $A=\left\{x \in X \mid T_{x}\right.$ is conjugate to $\left.T_{p}\right\}$ is equal to the image of (3). The fixed point set $X^{T_{p}}$ is closed in $X$, hence compact. Since $G$ is compact, the product $G \times X^{T_{p}}$ is compact, and the image of (3) is compact, hence closed. Thus $A$ is a closed subset of $X$. 
Since $X$ is compact and Hausdorff it is completely regular. By a theorem of Gleason [5, Theorem 3.3], $G$-orbits in $X$ admit local cross sections. In particular, for every $x \in X$ there is an open neighbourhood $x \in U \subseteq X$ such that for every $y \in U$, the stabilizer $G_{y}$ is a subgroup of a conjugate of $G_{x}$. Since $G_{x}$ and $G_{y}$ have the same rank, this implies that $T_{x}$ and $T_{y}$ are conjugate. It follows that $A$ is an open subset of $X$. Since $X$ is connected, it follows that $A=X$.

Lemma 3.3 Let $G$ act on $X$ from the left and let $x \in X^{T_{p}}$. Then $g \cdot x \in X^{T_{p}}$ if and only if $g \in N\left(T_{p}\right) G_{x}^{0}$, where $G_{x}^{0}$ is the identity component of the stabilizer $G_{x}$.

Proof If $g \cdot x \in X^{T_{p}}$, then $g^{-1} \operatorname{tg} \cdot x=x$ for all $t \in T_{p}$, so

$$
g^{-1} T_{p} g \subset G_{x} .
$$

Since stabilizers have constant rank, both $T_{p}$ and $g^{-1} T_{p} g$ are maximal in $G_{x}$, so for some $h \in G_{x}^{0}, h^{-1} g^{-1} T_{p} g h=T_{p}$, and thus $g \in N\left(T_{p}\right) G_{x}^{0}$. The other direction is clear.

Lemma 3.4 Let $\left(\phi: G \times X^{T_{p}} \rightarrow X, N\left(T_{p}\right)\right)$ be defined as above. For every $x \in X$, the orbit space $\phi^{-1}(x) / N\left(T_{p}\right) \cong G_{x}^{0} / N_{G_{x}^{0}}(H)$, where $H$ is a maximal torus in $G_{x}^{0}$.

Proof We may assume by equivariance that $x \in X^{T}$. We have isomorphisms of right $N\left(T_{p}\right)$-spaces

$$
\begin{aligned}
\phi^{-1}(x) & =\left\{(g, y) \in G \times X^{T_{p}} \mid g \cdot y=x\right\} \\
& =\left\{\left(g^{-1}, g \cdot x\right) \mid g \in N\left(T_{p}\right) G_{x}^{0}\right\} \cong G_{x}^{0} N\left(T_{p}\right),
\end{aligned}
$$

where the middle equality follows from the preceding lemma. It follows that

$$
\phi^{-1}(x) / N\left(T_{p}\right) \cong G_{x}^{0} N\left(T_{p}\right) / N\left(T_{p}\right) \cong G_{x}^{0} / N_{G_{x}^{0}}\left(T_{p}\right) .
$$

Proof of Proposition 3.1 Since both $G$ and $X$ are compact, it follows that $G \times X^{T_{p}}$ is compact and thus $\phi$ is closed. From Lemma 3.2, it follows that every $G$-orbit in $X$ must intersect $X^{T_{p}}$, so $\phi$ is surjective. Finally, the homeomorphism $\phi^{-1}(x) / N\left(T_{p}\right) \cong$ $G_{x}^{0} / N_{G_{x}^{0}}(H)$ from Lemma 3.4 implies that $H^{*}\left(\phi^{-1}(x) / N\left(T_{p}\right) ; F\right)$ is acyclic over fields of characteristic coprime to the order of the Weyl group, as explained in [2, Section 3].

Proof of Theorem 1.2 The map $G \times_{N\left(T_{p}\right)} X^{T_{p}} \rightarrow X$ is $G$-equivariant and a cohomology isomorphism, so it induces an isomorphism in equivariant cohomology

$$
H_{G}^{*}(X) \cong H_{G}^{*}\left(G \times_{N\left(T_{p}\right)} X^{T_{p}}\right) .
$$


The action of $N\left(T_{p}\right)$ on $G \times X^{T_{p}}$ is free and commutes with $G$, so we also have an isomorphism

$$
H_{G}^{*}\left(G \times N\left(T_{p}\right) X^{T_{p}}\right) \cong H_{G \times N\left(T_{p}\right)}^{*}\left(G \times X^{T_{p}}\right) .
$$

Finally, $G$ acts freely on $G \times X^{T_{p}}$, so we have an isomorphism

$$
H_{G \times N\left(T_{p}\right)}^{*}\left(G \times X^{T_{p}}\right) \cong H_{N\left(T_{p}\right)}^{*}\left(X^{T_{p}}\right) .
$$

\section{Formula for compact connected $G$}

Let $G$ be a compact connected Lie group and $\sigma \in \operatorname{Aut}(G)$. Let $G^{\sigma} \leq G$ denote the subgroup of elements fixed by $\sigma$, and let $T^{\sigma}$ be a maximal torus in (the identity component of) $G^{\sigma}$. Let $C\left(T^{\sigma}\right)=C_{G}\left(T^{\sigma}\right)$ and $N\left(T^{\sigma}\right)=N_{G}\left(T^{\sigma}\right)$ be the centralizer and normalizer of $T^{\sigma}$ in $G$ respectively. The twisted adjoint action restricts to an action of $N\left(T^{\sigma}\right)$ on $C\left(T^{\sigma}\right)$, which we denote by $C\left(T^{\sigma}\right)_{\mathrm{Ad}_{\sigma}}$. The goal of this section is to prove the following.

Proposition 4.1 Let $G$ be a compact connected Lie group and $\sigma \in \operatorname{Aut}(G)$ an automorphism such that some conjugate $g \sigma g^{-1}$ has finite order. Then there is a cohomology isomorphism

$$
H^{*}\left(B L_{\sigma} G\right) \cong H_{N\left(T^{\sigma}\right)}^{*}\left(C\left(T^{\sigma}\right)_{\operatorname{Ad}_{\sigma}}\right)
$$

for coefficient fields coprime to the order of the Weyl group of $G$.

The following result is not original (it follows implicitly from [13]), but I have not been able to find a clean statement in the literature.

Lemma 4.2 There is a natural homotopy equivalence $B L_{\sigma} G \cong E G \times_{G} G_{\mathrm{Ad}_{\sigma}}$.

Proof Consider the action of $L_{\sigma} G$ on the contractible based path space

$$
P G:=\operatorname{Maps}\left((I, 0),\left(G, \operatorname{Id}_{G}\right)\right)
$$

by $(\gamma \cdot x)(t)=\gamma(t) x \gamma(0)^{-1}$. Since $P G$ is contractible, the homotopy quotient $P G_{h L_{\sigma} G}$ is a model for $B L_{\sigma} G$.

The based loop group $\Omega G:=\left\{\gamma \in L_{\sigma} G \mid \gamma(0)=\gamma(1)=\operatorname{Id}_{G}\right\}$ acts freely on $P G$, so $P G_{h L_{\sigma} G}$ is equivalent to the homotopy quotient of the residual action of $L_{\sigma} G / \Omega G \cong$ $G$ on $P G / \Omega G \cong G_{\mathrm{Ad}_{\sigma}}$. 
The isomorphism class of $L_{\sigma} G$ depends only on the element of the outer automorphism group $\operatorname{Out}(G)=\operatorname{Aut}(G) / \operatorname{Inn}(A)$ represented by $\sigma$; see [9, Section 3.7]. Similarly, if $\sigma^{\prime}=\operatorname{Ad}_{h} \circ \sigma$ represent the same outer automorphism, then the map

$$
G_{\mathrm{Ad}_{\sigma}} \rightarrow G_{\mathrm{Ad}_{\sigma^{\prime}}}, \quad x \mapsto x h^{-1}
$$

in an isomorphism of $G$-spaces. Thus we may assume without loss of generality that $\sigma$ has finite order.

Lemma 4.3 Let $G$ be a compact connected Lie group. If $\sigma \in \operatorname{Aut}(G)$ has finite order then the twisted adjoint action of $G$ on $G_{\mathrm{Ad}_{\sigma}}$ has constant rank stabilizers.

Proof If $\sigma$ has order $n$, then it can be used to construct a semi-direct product $\mathbb{Z}_{n} \ltimes G$. It is explained in the introduction that the action of $G$ on $G_{\mathrm{Ad}_{\sigma}}$ is isomorphic to standard adjoint action of $G=\{0\} \times G$ on the path component $\{1\} \times G \cong G_{\operatorname{Ad}_{\sigma}}$.

The result now follows from a fundamental property of the adjoint action of discompact connected Lie groups found at the end of Chapter II of [11].

Proof of Proposition 4.1 By Lemma 4.2 we have $H^{*}\left(B L_{\sigma} G\right) \cong H_{G}^{*}\left(G_{\mathrm{Ad}_{\sigma}}\right)$. By Lemma 4.3 and Theorem 1.2 we have

$$
H_{G}^{*}\left(G_{\mathrm{Ad}_{\sigma}}\right) \cong H_{N\left(T_{p}\right)}^{*}\left(G_{\mathrm{Ad}_{\sigma}}^{T_{p}}\right)
$$

for any choice of $p \in G_{\mathrm{Ad}_{\sigma}}$. Choose $p=\operatorname{Id}_{G}$.

The stabilizer of the identity element $\operatorname{Id}_{G} \in G$ under the twisted adjoint action is exactly the subgroup $G^{\sigma}$ of elements invariant under $\sigma$. Let $T^{\sigma}$ denote a maximal torus of $G^{\sigma}$. The restriction of the twisted adjoint action to $T^{\sigma}$ agrees with the ordinary adjoint action. It follows that the set of $T^{\sigma}$-fixed points is precisely the centralizer $C\left(T^{\sigma}\right):=\left\{g \in G \mid g t=t g\right.$, for all $\left.t \in T^{\sigma}\right\}$. Then, as desired,

$$
H_{N\left(T_{p}\right)}^{*}\left(G_{\mathrm{Ad}_{\sigma}}^{T_{p}}\right) \cong H_{N\left(T^{\sigma}\right)}^{*}\left(C\left(T^{\sigma}\right)_{\mathrm{Ad}_{\sigma}}\right) .
$$

Example 1 If $\sigma \in \operatorname{Aut}(G)$ is the identity, we have $T^{\sigma}=T$ is a maximal torus with $N\left(T^{\sigma}\right)=N(T)$ acting on $C\left(T^{\sigma}\right)=T$ by the standard adjoint action. Proposition 4.1 gives us the formula

$$
H^{*}(B L G)=H_{G}^{*}(G) \cong H_{N(T)}^{*}(T) \cong\left(H^{*}(T) \otimes H^{*}(B T)\right)^{W}
$$

for coefficients coprime to the order of the Weyl group $W=N(T) / T$.

Remark 1 If $G$ is abelian, then $N\left(T^{\sigma}\right)=C\left(T^{\sigma}\right)=G$, so Proposition 4.1 offers no improvement over Lemma 4.2. The formula is more interesting in the opposite extreme when $G$ is semisimple, which is the subject of Theorem 1.1. 


\section{Proof of Theorem 1.1}

Assume throughout this section that $G$ is a compact connected semisimple Lie group, and that cohomology is taken with coefficient field $F$ of characteristic $p$ coprime to the orders of the Weyl group $W_{G}=N_{G}(T) / T$, of $[\sigma]$, and of $\pi_{0}\left(G^{\sigma}\right)$.

Lemma 5.1 Let $T^{\sigma}$ be a maximal torus in $G_{0}^{\sigma}$ and $C\left(T^{\sigma}\right)$ the centralizer of $T^{\sigma}$ in $G$. Then:

(a) $C\left(T^{\sigma}\right)=T$ is a maximal torus in $G$ and $T^{\sigma}=G_{0}^{\sigma} \cap T$.

(b) The restriction of $\sigma$ to $T$ preserves a Weyl chamber, and thus has finite order equal to that of the outer automorphism $[\sigma] \in \operatorname{Out}(G)$.

Proof This is mostly just a restatement of [11, Chapter II, Section 3, Proposition 2]. The only addition is that $\sigma_{T}$ has order equal to $[\sigma]$. This follows because if $[\sigma]$ has order $n$, then $\sigma^{n}$ is an inner automorphism that preserves a Weyl chamber of $T$ and thus must restrict to the identity map on $T$.

The twisted adjoint action restricts to the standard adjoint action for the subgroup $N_{G_{0}^{\sigma}}\left(T^{\sigma}\right) \subseteq N_{G}\left(T^{\sigma}\right)$ acting on the subspace $T^{\sigma} \subseteq T$, so these inclusions give rise to morphism in equivariant cohomology, which by Proposition 4.1 fits into a commutative diagram

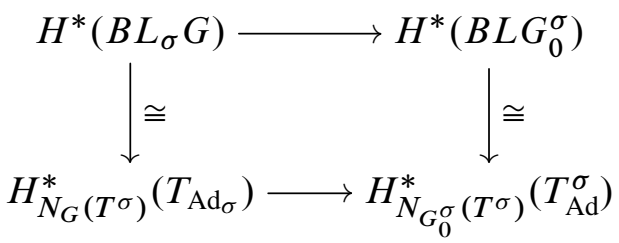

where the top arrow is the subject of Theorem 1.1.

Since $C\left(T^{\sigma}\right) \leq N_{G}\left(T^{\sigma}\right) \leq N_{G}\left(C\left(T^{\sigma}\right)\right)$, it follows from Lemma 5.1 that

$$
T \leq N_{G}\left(T^{\sigma}\right) \leq N_{G}(T) .
$$

Define $W_{\sigma}:=N_{G}\left(T^{\sigma}\right) / T$. Then $W_{\sigma} \subseteq W_{G}=N_{G}(T) / T$ is a finite group of order coprime to $p$. By Theorem 2.2, there is a natural isomorphism

$$
H_{N_{G}\left(T^{\sigma}\right)}^{*}\left(T_{\mathrm{Ad}_{\sigma}}\right) \cong H_{T}^{*}\left(T_{\mathrm{Ad}_{\sigma}}\right)^{W_{\sigma}} .
$$

The Weyl group $W_{G_{0}^{\sigma}}:=N_{G_{0}^{\sigma}}\left(T^{\sigma}\right) / T^{\sigma}$ acts faithfully on $T^{\sigma}$ by the adjoint action, so the inclusion $N_{G_{0}^{\sigma}}\left(T^{\sigma}\right) \hookrightarrow N_{G}\left(T^{\sigma}\right)$ descends to an injection $W_{G_{0}^{\sigma}} \hookrightarrow W_{\sigma}$. It follows from Lagrange's theorem that the order of $W_{G_{0}^{\sigma}}$ is coprime to $p$. We gain a natural isomorphism

$$
H_{N_{G_{0}^{\sigma}}\left(T^{\sigma}\right)}^{*}\left(T_{\mathrm{Ad}}^{\sigma}\right) \cong H_{T^{\sigma}}^{*}\left(T_{\mathrm{Ad}}^{\sigma}\right)^{W_{G_{0}^{\sigma}}^{\sigma}}
$$


These natural isomorphisms permit us to replace (9) with the commuting diagram

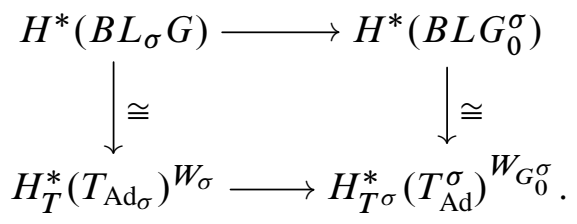

Lemma 5.2 The inclusion $T^{\sigma} \subseteq T$ induces an isomorphism in equivariant cohomology

$$
H_{T}^{*}\left(T_{\mathrm{Ad}_{\sigma}}\right) \cong H_{T^{\sigma}}^{*}\left(T_{\mathrm{Ad}}^{\sigma}\right) .
$$

Proof The action of $T^{\sigma}$ on $T_{\mathrm{Ad}}^{\sigma}$ is trivial, so the homotopy quotient is the product $B T^{\sigma} \times T^{\sigma}$ and the equivariant cohomology ring is

$$
H_{T^{\sigma}}^{*}\left(T_{\mathrm{Ad}}^{\sigma}\right) \cong H^{*}\left(B T^{\sigma}\right) \otimes H^{*}\left(T^{\sigma}\right) .
$$

The twisted adjoint action of $t \in T$ on $x \in T_{\mathrm{Ad}_{\sigma}}$

$$
\operatorname{Ad}_{\sigma}(t)(x)=t x \sigma(t)^{-1}=t \sigma(t)^{-1} x
$$

is simply translation by $t \sigma(t)^{-1}$. Consequently, $T$ acts on $T_{\mathrm{Ad}_{\sigma}}$ with constant stabilizer $G^{\sigma} \cap T$.

Choose a complementary subtorus $T^{\prime}$ so that $T=T^{\sigma} \times T^{\prime}$. Then the factor $T^{\sigma}$ acts trivially on $T_{\operatorname{Ad}_{\sigma}}$, so the homotopy quotient satisfies

$$
\left(T_{\mathrm{Ad}_{\sigma}}\right)_{h T} \cong B T^{\sigma} \times\left(T_{\mathrm{Ad}_{\sigma}}\right)_{h T^{\prime}},
$$

where in the second factor we consider the restricted action of $T^{\prime}$ on $T_{\mathrm{Ad}_{\sigma}}$ which has constant stabilizer $T^{\prime} \cap G^{\sigma}$. It follows that the projection map onto the orbit space

$$
\left(T_{\mathrm{Ad}_{\sigma}}\right)_{h T^{\prime}} \rightarrow T_{\mathrm{Ad}_{\sigma}} / T^{\prime}=T_{\mathrm{Ad}_{\sigma}} / T
$$

has homotopy fibre $B\left(T^{\prime} \cap G^{\sigma}\right)$.

Observe that the induced homomorphism $T^{\prime} \cap G^{\sigma} \rightarrow \pi_{0}\left(G^{\sigma}\right)$ is injective, because $T^{\prime} \cap G_{0}^{\sigma}=T^{\prime} \cap T^{\sigma}=\left\{\operatorname{Id}_{G}\right\}$. In particular, the order of $T^{\prime} \cap G^{\sigma}$ divides the order of $\pi_{0}\left(G^{\sigma}\right)$, so $B\left(T^{\prime} \cap G^{\sigma}\right)$ is acyclic over the field $F$ and

$$
H_{T}^{*}\left(T_{\mathrm{Ad}_{\sigma}}\right) \cong H^{*}\left(B T^{\sigma}\right) \otimes H_{T^{\prime}}^{*}\left(T_{\mathrm{Ad}_{\sigma}}\right) \cong H^{*}\left(B T^{\sigma}\right) \otimes H^{*}\left(T_{\mathrm{Ad}_{\sigma}} / T\right) .
$$

The result now follows from Lemma 5.3.

Lemma 5.3 The map $\phi: T^{\sigma} \rightarrow T_{\mathrm{Ad}_{\sigma}} / T$ obtained by composing inclusion and quotient maps is a covering map of finite degree coprime to $p$. In particular, $\phi$ induces a cohomology isomorphism in characteristic $p$. 
Proof Let $n$ be the order of $\left.\sigma\right|_{T}$. By Lemma $5.1 n$ is coprime to $p$.

As explained in the proof of Lemma 5.2, the twisted adjoint action of $t \in T$ on $T_{\operatorname{Ad}_{\sigma}}$ is simply translation by $t \sigma(t)^{-1}$. The orbit space $T_{\mathrm{Ad}_{\sigma}} / T$ may thus be identified with the coset space $T / H$ where $H:=\left\{t \sigma(t)^{-1} \mid t \in T\right\}$, and $\phi$ can be identified with the corresponding group homomorphism

$$
\phi^{\prime}: T^{\sigma} \rightarrow T / H
$$

Since $\phi^{\prime}$ is a homomorphism between tori of equal rank, it is enough to show that $\operatorname{ker}\left(\phi^{\prime}\right)=T^{\sigma} \cap H$ has finite order dividing a power of $n$.

Suppose that $t \in H \cap T^{\sigma}$. Then both $t=\sigma(t)$ and $t=s \sigma(s)^{-1}$ for some $s \in T$. Thus

$$
t^{n}=t \sigma(t) \sigma^{2}(t) \cdots \sigma^{n-1}(t)=\left(s \sigma(s)^{-1}\right) \cdots\left(\sigma^{n-1}(s) \sigma^{n}(s)^{-1}\right)=s \sigma^{n}(s)^{-1}=\operatorname{Id}_{T},
$$

so $\operatorname{ker}\left(\phi^{\prime}\right)$ is a subgroup of $T_{n}:=\left\{t \in T \mid t^{n}=\operatorname{Id}_{T}\right\}$, which is a group of order $n^{\operatorname{rank}(T)}$. The result follows by Lagrange's theorem.

Next, we want to understand the $W_{\sigma}$ and $W_{G_{0}^{\sigma}}$ actions. Observe that the residual $W_{G_{0}^{\sigma}}$-action on the homotopy quotient $\left(T_{\mathrm{Ad}}\right)_{h T^{\sigma}}=B T^{\sigma} \times T^{\sigma}$ acts diagonally in the standard way on each factor, so the action extends to $W_{\sigma}=N_{G}\left(T^{\sigma}\right) / T$ in the standard way.

Lemma 5.4 The isomorphism $H_{T \sigma}^{*}\left(T_{\mathrm{Ad}}^{\sigma}\right) \cong H_{T}^{*}\left(T_{\mathrm{Ad}_{\sigma}}\right)$ defined in Lemma 5.2 is $W_{\sigma}$-equivariant with respect to the actions defined above.

Proof Both actions are diagonal with respect to the Kunneth factorizations defined in the proof of Lemma 5.2:

$$
\begin{aligned}
& H_{T^{\sigma}}^{*}\left(T_{\mathrm{Ad}}^{\sigma}\right) \cong H^{*}\left(B T^{\sigma}\right) \otimes H^{*}\left(T^{\sigma}\right), \\
& H_{T}^{*}\left(T_{\mathrm{Ad}_{\sigma}}\right) \cong H^{*}\left(B T^{\sigma}\right) \otimes H^{*}\left(T_{\mathrm{Ad}_{\sigma}} / T\right) .
\end{aligned}
$$

The action on the first factors are the same, since the group $T^{\sigma}$ acts trivially in both cases. It remains to consider action on the second factors are equivariant with respect to the isomorphism $H^{*}\left(T^{\sigma}\right) \cong H^{*}\left(T_{\mathrm{Ad}_{\sigma}} / T\right)$ from Lemma 5.3.

Let $\phi: T_{\mathrm{Ad}}^{\sigma} \rightarrow T_{\mathrm{Ad}_{\sigma}} / T$ be the covering map, $n \in N_{G}\left(T^{\sigma}\right)$, and $x \in T_{\mathrm{Ad}}^{\sigma}$. Then

$$
\phi \circ \operatorname{Ad}(n)(x)=\phi\left(n x n^{-1}\right)=\left[n x n^{-1}\right]
$$

while

$$
\operatorname{Ad}_{\sigma}(n) \circ \phi(x)=\left[n x \sigma\left(n^{-1}\right)\right]=\left[n x n^{-1} n \sigma\left(n^{-1}\right)\right] .
$$

The two maps $\phi \circ \operatorname{Ad}(n)$ and $\operatorname{Ad}_{\sigma}(n) \circ \phi$ agree up to translation by $n \sigma\left(n^{-1}\right) \in T$, so they are homotopic and define the same map on cohomology. 
Proof of Theorem 1.1 Consider again the diagram (10). It follows from Lemma 5.2 that the horizontal arrows are injective, and from Lemma 5.4 that the image of the bottom arrow is equal to

$$
H_{T^{\sigma}}^{*}\left(T_{\mathrm{Ad}}^{\sigma}\right)^{W_{\sigma}} \subseteq H_{T^{\sigma}}^{*}\left(T_{\mathrm{Ad}}^{\sigma}\right)^{W_{G_{0}^{\sigma}}^{\sigma}}
$$

\section{Simplifying the calculations}

It can be tricky to apply Theorem 1.1 directly, because it requires an explicit understanding of $W_{\sigma}$ and its action on $H^{*}\left(B L G_{0}^{\sigma}\right)$. Fortunately, matters simplify under certain conditions.

Throughout this section let $G$ be a compact connected Lie group, let $\sigma \in \operatorname{Aut}(G)$ be an automorphism, and let $T \leq G$ be a maximal torus containing a maximal torus $T^{\sigma} \leq G_{0}^{\sigma}$. There is a natural action of $\operatorname{Aut}\left(G_{0}^{\sigma}\right)$ on $B L G_{0}^{\sigma}$. By a result of Segal [10, Section 3], inner automorphisms act by isotopy, so we obtain a natural action of $\operatorname{Out}\left(G_{0}^{\sigma}\right)$ on $H^{*}\left(B L G_{0}^{\sigma}\right)$.

Proposition 6.1 Suppose that the adjoint action of $W_{\sigma}$ on $T^{\sigma}$ consists of transformations that extend to automorphisms of $G_{0}^{\sigma}$. Then the $W_{\sigma}$-action on $H^{*}\left(B L G_{0}^{\sigma}\right)$ factors through the $\operatorname{Out}\left(G_{0}^{\sigma}\right)$-action.

Proof The injection $H^{*}\left(B L G_{0}^{\sigma}\right) \hookrightarrow H_{T^{\sigma}}^{*}\left(T^{\sigma}\right)$ in (10) is equivariant with respect to automorphisms of $G_{0}^{\sigma}$ which preserve $T^{\sigma}$. Thus if every transformation of $T^{\sigma}$ extends, it follows that

$$
H^{*}\left(B L G_{0}^{\sigma}\right)^{W_{\sigma}}=H^{*}\left(B L G_{0}^{\sigma}\right)^{\Gamma}
$$

for some subset $\Gamma \subseteq \operatorname{Out}\left(G_{0}^{\sigma}\right)$. An automorphism of a maximal torus extends to at most one outer automorphism of its compact connected Lie group, so $\Gamma$ is the image of a well-defined homomorphism $W_{\sigma} \rightarrow \operatorname{Out}\left(G_{0}^{\sigma}\right)$.

Remark 2 The $W_{\sigma}$-action on $T^{\sigma}$ doesn't always extend to automorphisms of $G_{0}^{\sigma}$. For example, let $G=\mathrm{SU}(3)$ and $\sigma=\operatorname{Ad}_{g}$, where

$$
g=\left(\begin{array}{rrr}
1 & 0 & 0 \\
0 & -1 & 0 \\
0 & 0 & -1
\end{array}\right)
$$

Then $T^{\sigma}=T$, so $W_{\sigma}=W \cong \mathrm{S}_{3}$, but the $W$-action does not extend to $G^{\sigma} \cong U(2)$ because it does not preserve its root system. 
We can use root systems to check whether the $W_{\sigma}$-action extends to $G_{0}^{\sigma}$. Let $\mathfrak{g}, \mathfrak{g}^{\sigma}$, $\mathfrak{t}$, and $\mathfrak{t}^{\sigma}$ denote the complexified Lie algebras of $G, G_{0}^{\sigma}, T$, and $T^{\sigma}$ respectively. The root system $\Phi \subset \mathfrak{t}^{*}$ is simply the set of weights of the $\mathfrak{t}$-module $\mathfrak{g} / \mathfrak{t}$ under the adjoint action. Similarly, the root system of $\Phi_{\sigma} \subset\left(\mathfrak{t}^{\sigma}\right)^{*}$ is the set of weights of the $\mathfrak{t}^{\sigma}$-module $\mathfrak{g}^{\sigma} / \mathfrak{t}^{\sigma}$. The inclusion $\mathfrak{t}^{\sigma} \hookrightarrow \mathfrak{t}$ determines a projection map $\pi: \mathfrak{t}^{*} \rightarrow\left(\mathfrak{t}^{\sigma}\right)^{*}$. The natural injection of $\mathfrak{t}^{\sigma}$-modules, $\mathfrak{g}^{\sigma} / \mathfrak{t}^{\sigma} \subseteq \mathfrak{g} / \mathfrak{t}$, implies that $\Phi_{\sigma} \subseteq \pi(\Phi)$.

Corollary 6.2 If the automorphism groups of $\pi(\Phi)$ and $\Phi_{\sigma}$ coincide, then $W_{\sigma}$ acts on $H^{*}\left(B L G_{0}^{\sigma}\right)$ via $\operatorname{Out}\left(G_{0}^{\sigma}\right)$.

Proof Since compact Lie groups can be constructed functorially from their root system and weight lattice, it will suffice to prove that the action of $W_{\sigma}$ on $T^{\sigma}$ preserves the root system $\Phi_{\sigma}$.

The action of $W_{\sigma}$ on $\mathfrak{t}$ is a restriction of the standard Weyl group action, so it clearly preserves the root system $\Phi \subset \mathfrak{t}^{*}$. The projection map $\pi: \mathfrak{t}^{*} \rightarrow\left(\mathfrak{t}^{\sigma}\right)^{*}$ is $W_{\sigma}$-equivariant, so $W_{\sigma}$ also preserves $\pi(\Phi)$. Since by hypothesis, the automorphisms of $\pi(\Phi)$ and $\Phi_{\sigma}$ coincide, $W_{\sigma}$ must also preserve $\Phi_{\sigma}$.

A sufficient condition for the hypothesis of Corollary 6.2 to hold is that $\pi(\Phi)=\Phi_{\sigma}$. We have an easy-to-check criterion for this.

Corollary 6.3 The automorphism $\sigma$ induces a permutation of the root system $\Phi$ of $(G, T)$. If the number of $\sigma$-orbits in $\Phi$ is equal to the number of roots in $\Phi_{\sigma}$, then $W_{\sigma}$ acts on $H^{*}\left(B L G_{0}^{\sigma}\right)$ via $\operatorname{Out}\left(G_{0}^{\sigma}\right)$.

Proof Because we have an inclusion $\Phi_{\sigma} \subseteq \pi(\Phi)$, it suffices to show that $\pi(\Phi)$ has cardinality equal to $\Phi_{\sigma}$. Since any two roots in $\Phi$ lying in the same $\sigma$-orbit are sent to the same element of $\pi(\Phi)$, the result follows.

Remark 3 We know from Lemma 5.1 that $\sigma$ preserves a Weyl chamber of $T$. Hence it also preserves a set positive roots for $\Phi$ and thus the action of $\sigma$ on roots is determined by an automorphism of the Dynkin diagram. We will use this point of view to count orbits in concrete examples in the following section.

\section{Examples}

In this section, we compute $H^{*}\left(B L_{\sigma} G ; F\right)$ in several examples, including all automorphisms of simple Lie groups. By the following argument, it makes little difference which finite cover of the adjoint group we work with. 
Proposition 7.1 Let $\phi: G \rightarrow G^{\prime}$ be a surjective homomorphism of compact connected Lie groups with finite kernel $K$, and let $\sigma \in \operatorname{Aut}(G)$ descend to $\sigma^{\prime} \in \operatorname{Aut}\left(G^{\prime}\right)$. Then the induced map on twisted loop groups determines a cohomology isomorphism

$$
H^{*}\left(B L_{\sigma} G\right) \cong H^{*}\left(B L_{\sigma^{\prime}} G^{\prime}\right)
$$

for coefficient fields of characteristic coprime to the order of $K$.

Proof For any $G^{\prime}$-space $X$, we may compose with $\phi$ to make $X$ into a $G$-space and resulting map of homotopy quotient

$$
E G \times{ }_{G} X \rightarrow E G^{\prime} \times_{G^{\prime}} X
$$

has homotopy fibre $B K$. Since $B K$ is acyclic over the coefficient field $F$, this means that $H_{G}^{*}(X) \cong H_{G^{\prime}}^{*}(X)$ for any $G^{\prime}$-space $X$ and in particular

$$
H_{G}^{*}\left(G_{\mathrm{Ad}_{\sigma^{\prime}}}^{\prime}\right) \cong H_{G^{\prime}}^{*}\left(G_{\mathrm{Ad}_{\sigma^{\prime}}}^{\prime}\right) .
$$

The map $\phi$ is also a covering map with deck transformation group $K$ acting transitively on the fibres. The transfer map determines an isomorphism

$$
H^{*}(G)^{K} \cong H^{*}\left(G^{\prime}\right)
$$

for fields of characteristic coprime to \# $K$. Since the deck transformations are isotopies of $G$, they act trivially on cohomology and $\phi$ is a cohomology isomorphism

$$
H^{*}(G) \stackrel{\phi}{\cong} H^{*}\left(G^{\prime}\right)
$$

and similarly for equivariant cohomology

$$
H_{G}^{*}\left(G_{\mathrm{Ad}_{\sigma}}\right) \stackrel{\phi}{\cong} H_{G}^{*}\left(G_{\mathrm{Ad}_{\sigma}}^{\prime}\right)
$$

\section{A Untwisted loop groups}

The following proposition is well known (see, for example, Kuribayashi, Mimura, Nishimoto [6, Theorem 1.2]), but I include a proof both for convenience and because I have not found a statement of the result in this generality in the literature.

Proposition 7.2 Let $G$ be a compact connected Lie group. For any field $F$ of characteristic $p$ such that $H^{*}(G ; \mathbb{Z})$ is $p$-torsion free, we have

$$
H^{*}(B L G ; F) \cong H^{*}(G) \otimes H^{*}(B G) \cong \Lambda\left(x_{1}, \ldots, x_{r}\right) \otimes F\left[y_{1}, \ldots, y_{r}\right],
$$

where $r$ equals the rank of $G$ and the degrees of the generators are independent of $F$. 
Remark 4 A sufficient condition for $H^{*}(G ; \mathbb{Z})$ to be $p$-torsion free is for $p$ to be coprime to the order of the Weyl group of $G$. We refer to Borel [4] for the degrees of the generators for various simple groups $G$.

Proof Under the hypotheses above, we have isomorphisms

$$
H^{*}(G ; F) \cong \Lambda\left(x_{1}, \ldots, x_{r}\right),
$$

an exterior algebra where $r$ and the odd degrees $\operatorname{deg}\left(x_{i}\right)$ are independent of $p$, and

$$
H^{*}(B G ; F) \cong F\left[y_{1}, \ldots, y_{r}\right]
$$

such that $\operatorname{deg}\left(y_{i}\right)=\operatorname{deg}\left(x_{i}\right)+1$; see [4, Section 9].

The classifying space $B L G=E G \times{ }_{G} G_{\mathrm{Ad}}$, fits into a fibration sequence

$$
G \stackrel{i}{\rightarrow} B L G \rightarrow B G
$$

which has Serre spectral sequence $\mathrm{E}_{2}=H^{*}(B G ; F) \otimes H^{*}(G ; F)$ converging to $H^{*}(B L G ; F)$. This spectral sequence is known to collapse for $F=\mathbb{Q}$ (see [2], for example) and thus by the universal coefficient theorem must collapse for all $F$ under consideration, proving that (11) holds as an isomorphism of $H^{*}(B G ; F)$-modules. Since $H^{*}(G ; F)=\Lambda\left(x_{1}, \ldots, x_{r}\right)$ is free as a supercommutative algebra, we can upgrade (11) to an algebra isomorphism using the Leray-Hirsch theorem to lift the generators of $x_{1}, \ldots, x_{r}$ via the surjection $i^{*}: H^{*}(B L G ; F) \rightarrow H^{*}(G ; F)$.

\section{B SU(n) with entry-wise complex conjugation}

Let $\sigma \in \operatorname{Aut}(\mathrm{SU}(n))$ denote matrix entry-wise complex conjugation. For $n \geq 3$, $[\sigma]$ generates the outer automorphism group $\operatorname{Out}(\mathrm{SU}(n)) \cong \mathbb{Z}_{2}$. The fixed point set $\mathrm{SU}(n)^{\sigma}=\mathrm{SO}(n)$. Observe that $\sigma(A)^{-1}=A^{T}$ where $A^{T}$ denotes the transpose, so the twisted adjoint action is

$$
\operatorname{Ad}_{\sigma}(A)(X)=A X \sigma(A)^{-1}=A X A^{T},
$$

which may be interpreted as a change of basis operation for a bilinear form (see Remark 6).

Proposition 7.3 For coefficient fields $F$ coprime to $n$ !, the inclusion $\mathrm{SO}(n) \hookrightarrow \mathrm{SU}(n)$ determines an isomorphism

$$
H^{*}\left(B L_{\sigma} \mathrm{SU}(n) ; F\right) \cong H^{*}(B L \mathrm{SO}(n) ; F)^{\mathbb{Z}_{2}},
$$

where $\mathbb{Z}_{2}$ acts on $\mathrm{SO}(n)$ by an orientation reversing change of basis. 
Proof For $n=1,2$, we have $T^{\sigma}=G_{0}^{\sigma}$, so Proposition 6.1 applies immediately. For $n \geq 3$, we must study the action of $\sigma$ on the root system $\Phi$ of SU(n) in order to apply Corollary 6.2. The roots of $\mathrm{SU}(n)$ are $e_{i}-e_{j}$ for $i, j \in\{1, \ldots, n\}, i \neq j$, and the automorphism induces the involution of root

$$
\sigma\left(e_{i}-e_{j}\right)=e_{n+1-j}-e_{n+1-i} .
$$

Since $\sigma$ has order two, the projection map satisfies

$$
\pi(x)=\frac{x+\sigma(x)}{2} .
$$

For $i<(n+1) / 2$, define

$$
E_{i}=-E_{n+1-i}=\frac{1}{2}\left(e_{i}-e_{n+1-i}\right) .
$$

If $n$ is even, then for $i \neq j$

$$
\pi\left(e_{i}-e_{j}\right)= \begin{cases} \pm E_{i} \pm E_{j} & \text { if } i+j \neq n+1 \\ \pm 2 E_{i} & \text { if } i+j=n+1\end{cases}
$$

which is exactly the root system $C_{n / 2}$. If $n$ is odd, then for $i \neq j$

$$
\pi\left(e_{i}-e_{j}\right)= \begin{cases} \pm E_{i} \pm E_{j} & \text { if } i+j \neq n+1 \text { and } \frac{n+1}{2} \notin\{i, j\} \\ \pm E_{i} & \text { if } j=\frac{n+1}{2} \\ \pm E_{j} & \text { if } i=\frac{n+1}{2} \\ \pm 2 E_{i} & \text { if } i+j=n+1\end{cases}
$$

which has the same automorphism group as $C_{(n-1) / 2}$. In both cases, the root system $\Phi_{\sigma}$ of $\operatorname{SO}(n)$ is the complement of $\left\{ \pm 2 E_{i} \mid i=1, \ldots,[n / 2]\right\}$ in $\pi(\Phi)$ and the automorphism group $\Phi_{\sigma}$ agrees with that of $\pi(\Phi)$ (both being equal to the automorphism group of the root lattice of $\mathrm{SO}(n))$. It follows from Corollary 6.2 that the $W_{\sigma}$-action on $H^{*}(B L \mathrm{SO}(n))$ is induced by outer automorphisms of $\mathrm{SO}(n)$.

If $n$ is odd, the outer automorphism group of $\mathrm{SO}(n)$ is trivial, so by Theorem 1.1

$$
H^{*}\left(B L_{\sigma} \mathrm{SU}(n)\right) \cong H^{*}(B L \mathrm{SO}(n)) .
$$

Moreover, an orientation reversing change of basis must be an inner automorphism of $\mathrm{SO}(n)$, hence also of $L \mathrm{SO}(n)$, thus it acts by an isotopy of $B L \mathrm{SO}(n)$ [10, Section 3], so $\mathbb{Z}_{2}$ acts trivially on cohomology and the result follows.

In case $n$ is even, the outer automorphism group of $\mathrm{SO}(n)$ is $\mathbb{Z}_{2}$ and is generated by orientation reversing change of basis. Let $P \in O(n)$ be an orientation reversing change of basis matrix. Then $i P \in \mathrm{SU}(n)$ and for any $X \in \mathrm{SO}(n)$,

$$
P X P^{-1}=(i P) X(i P)^{-1} \text {. }
$$


Thus the change of basis is induced by conjugation by an element of $\mathrm{SU}(n)$; the result now follows from Theorem 1.1

The twisted action (12) extends naturally to a twisted action of $U(n)$ on $U(n)$.

Proposition 7.4 The standard inclusion $\mathrm{SU}(n) \hookrightarrow U(n)$ induces a cohomology isomorphism

$$
H^{*}\left(B L_{\sigma} U(n) ; F\right) \cong H^{*}\left(B L_{\sigma} \mathrm{SU}(n) ; F\right)
$$

for coefficient fields $F$ of characteristic coprime to both 2 and $n$.

Proof Consider the surjective group homomorphism

$$
\phi: U(1) \times \mathrm{SU}(n) \rightarrow U(n), \quad(\lambda, A) \mapsto \lambda A,
$$

which has finite kernel $\mathbb{Z}_{n}$. The homomorphism is equivariant with respect to entry-wise complex conjugation, so by Proposition 7.1.

$$
H_{U(1) \times \mathrm{SU}(n)}^{*}\left((U(1) \times \mathrm{SU}(n))_{\mathrm{Ad}_{\sigma}}\right) \cong H_{U(n)}^{*}\left(U(n)_{\mathrm{Ad}_{\sigma}}\right) .
$$

Moreover, we have isomorphisms

$$
\begin{aligned}
H_{U(1) \times \mathrm{SU}(n)}^{*}\left((U(1) \times \mathrm{SU}(n))_{\mathrm{Ad}_{\sigma}}\right) & \cong H_{U(1)}^{*}\left(U(1)_{\mathrm{Ad}_{\sigma}}\right) \otimes H_{\mathrm{SU}(n)}^{*}\left(\mathrm{SU}(n)_{\mathrm{Ad}_{\sigma}}\right) \\
& \cong H^{*}\left(B \mathbb{Z}_{2}\right) \otimes H_{\mathrm{SU}(n)}^{*}\left(\mathrm{SU}(n)_{\mathrm{Ad}_{\sigma}}\right) \\
& \cong H_{\mathrm{SU}(n)}^{*}\left(\mathrm{SU}(n)_{\mathrm{Ad}_{\sigma}}\right)
\end{aligned}
$$

because the twisted $U(1)$-action on $U(1)_{\mathrm{Ad}_{\sigma}}$ is transitive with stabilizer $\mathbb{Z}_{2}$, and $B \mathbb{Z}_{2}$ is acyclic over $F$.

Corollary 7.5 Let $F$ be a field of characteristic coprime to $n$ ! and let $n=2 m$ or $n=2 m+1$. The standard inclusion of groups

$$
L O(n) \hookrightarrow L_{\sigma} U(n) \hookleftarrow L_{\sigma} \mathrm{SU}(n)
$$

induces isomorphisms

$$
\begin{aligned}
H^{*}(B L O(n) ; F) & \cong H^{*}\left(B L_{\sigma} U(n) ; F\right) \cong H^{*}\left(B L_{\sigma} \mathrm{SU}(n) ; F\right) \\
& \cong \Lambda\left(x_{3}, x_{7}, \ldots, x_{4 m-1}\right) \otimes S\left(y_{4}, \ldots, y_{4 m}\right),
\end{aligned}
$$

where the subscripts indicate the degrees of the generators.

Proof The loop group $L \mathrm{SO}(n)$ sits inside $L O(n)$ as an index-two subgroup, so $H^{*}(B L O(n)) \cong H^{*}(B L \mathrm{SO}(n))^{\mathbb{Z}_{2}}$. The result now follows from Propositions 7.4 and 7.3. 
Remark 5 Corollary 7.5 stands in contrast with the formula

$$
\begin{aligned}
H^{*}\left(B L_{\sigma} U(n) ; \mathbb{Z}_{2}\right) & \cong H^{*}\left(B L U(n) ; \mathbb{Z}_{2}\right) \\
& \cong \Lambda\left(x_{1}, x_{3}, \ldots, x_{2 n-1}\right) \otimes S\left(y_{2}, y_{4}, \ldots, y_{2 n}\right)
\end{aligned}
$$

derived by Baird [3].

Remark 6 The twisted action of $U(n)$ on $U(n)$ is homotopy equivalent to the change of basis action of $\mathrm{GL}_{n}(\mathbb{C})$ on the space of (not necessarily symmetric) non-degenerate bilinear forms on $\mathbb{C}^{n}$. Thus Corollary 7.5 also calculates the cohomology of the topological moduli stack of rank $n$ non-degenerate bilinear forms over $\mathbb{C}$.

\section{C $\operatorname{SO}(2 n)$ with orientation reversing change of basis}

The Weyl group of $\mathrm{SO}(2 n)$ has order $2^{n-1} n !$. An orientation reversing change of basis determines an automorphism $\sigma \in \operatorname{Aut}(\mathrm{SO}(2 n))$ of order two, which generates $\operatorname{Out}(\mathrm{SO}(2 n))$ for $n \geq 5$. The corresponding twisted loop group $L_{\sigma} \mathrm{SO}(2 n)$ can be understood as the gauge group of orthogonal, orientation preserving gauge transformations of a non-orientable $\mathbb{R}^{n}$-bundle over $S^{1}$.

Proposition 7.6 The block sum inclusion $\mathrm{SO}(2 n-1) \hookrightarrow \mathrm{SO}(2 n)$ induces a cohomology isomorphism

$$
\begin{aligned}
H^{*}\left(B L_{\sigma} \mathrm{SO}(2 n) ; F\right) & \cong H^{*}(B L \mathrm{SO}(2 n-1) ; F) \\
& \cong \Lambda\left(x_{3}, x_{7}, \ldots, x_{4 n-5}\right) \otimes S\left(y_{4}, \ldots, y_{4 n-4}\right)
\end{aligned}
$$

for coefficient field $F$ of odd characteristic coprime to $n !$.

Proof Set

$$
\sigma(A)=P A P^{-1}
$$

where

$$
P=\left(\begin{array}{cc}
\operatorname{Id}_{2 n-1} & 0 \\
0 & -1
\end{array}\right) .
$$

Then $\mathrm{SO}(2 n)^{\sigma}$ is isomorphic to $O(2 n-1)$ by the injection

$$
O(2 n-1) \hookrightarrow \operatorname{SO}(2 n), \quad B \mapsto\left(\begin{array}{cc}
B & 0 \\
0 & \operatorname{det}(B)
\end{array}\right),
$$

which has identity component isomorphic to $\mathrm{SO}(2 n-1)$.

For $n=1$, we have $\mathrm{SO}(2) \cong U(1)$ so this case has already been covered by Corollary 7.5. 
For $n \geq 2$, the root system of $\operatorname{SO}(2 n)$ consists of vectors $\pm\left(e_{i} \pm e_{j}\right)$ for $i \neq j$ in $\{1, \ldots, n\}$. The involution fixes $e_{i}$ for $i<n$ and sends $e_{n}$ to $-e_{n}$. One easily checks that there are $4\left(\begin{array}{l}n \\ 2\end{array}\right)-2(n-1)=2(n-1)^{2}$ which equals the number of roots of the fixed point subgroup $\mathrm{SO}(2 n-1)$. Thus by Corollary $6.3, W_{\sigma}$-action on $H^{*}(B L \mathrm{SO}(2 n-1))$ is induced by outer automorphisms of $\mathrm{SO}(2 n-1)$. The outer automorphism group of $\mathrm{SO}(2 n-1)$ is trivial, so the result follows by Theorem 1.1.

\section{D SO(8) with the triality automorphism}

The outer automorphism group of $\mathrm{SO}(8)$ is the permutation group $\mathrm{S}_{3}$. The order three automorphisms are represented by the triality automorphisms $\sigma, \sigma^{2} \in \operatorname{Aut}(\mathrm{SO}(8))$ which are related to realization of $\mathrm{SO}(8)$ as orthogonal transformations of the underlying vector space of the octonions; see Baez [1]. The fixed point set $\mathrm{SO}(8)^{\sigma}$ is equal to the automorphism group of the octonions $\mathrm{G}_{2}$.

The root system of $\mathrm{SO}(8)$ has 12 positive roots. The triality automorphism determines six orbits: three of order one and three of order three. Since the root system of $\mathrm{G}_{2}$ has 6 positive roots, Corollary 6.3 implies that $W_{\sigma}$ acts via outer automorphisms of $\mathrm{G}_{2}$. Since $\mathrm{G}_{2}$ has trivial outer automorphism group and they Weyl group of $\mathrm{SO}(8)$ has order $3 \cdot 2^{6}$, we conclude the following from Theorem 1.1 .

Proposition 7.7 Suppose $\sigma \in \operatorname{Aut}(\mathrm{SO}(8))$ is a triality automorphism. We have

$$
\begin{aligned}
H^{*}\left(B L_{\sigma} \mathrm{SO}(8) ; F\right) & \cong H^{*}\left(B L_{\sigma} \mathrm{SO}(8) ; F\right) \\
& \cong H^{*}\left(B L \mathrm{G}_{2} ; F\right) \cong \Lambda\left(x_{3}, x_{11}\right) \otimes F\left[y_{4}, y_{12}\right]
\end{aligned}
$$

for a coefficient field $F$ of characteristic coprime to 6 .

\section{E E6 with involution}

The outer automorphism group of a compact, simply connected group of type $\mathrm{E}_{6}$ is generated by an automorphism $\sigma$ of order two. The induced action on the set of positive roots has 24 orbits. The fixed point set $\mathrm{E}_{6}^{\sigma}$ is isomorphic to $\mathrm{F}_{4}$ which has 24 positive roots, so Corollary 6.3 applies. The Weyl group of $\mathrm{E}_{6}$ has order $51840=2^{7} 3^{4} 5$. Since $\mathrm{F}_{4}$ has trivial outer automorphism group, the following proposition follows from Theorem 1.1.

Proposition 7.8 Suppose $\sigma \in \operatorname{Aut}\left(\mathrm{E}_{6}\right)$ is not an inner automorphism. Then we have a cohomology isomorphism

$$
H^{*}\left(B L_{\sigma} \mathrm{E}_{6} ; F\right) \cong H^{*}\left(B L \mathrm{~F}_{4} ; F\right) \cong \Lambda\left(x_{3}, x_{11}, x_{15}, x_{23}\right) \otimes F\left[y_{4}, y_{12}, y_{16}, y_{24}\right]
$$

for coefficient fields $F$ of characteristic coprime to 30 . 


\section{References}

[1] J C Baez, The octonions, Bull. Amer. Math. Soc. 39 (2002) 145-205 MR1886087

[2] T J Baird, Cohomology of the space of commuting n-tuples in a compact Lie group, Algebr. Geom. Topol. 7 (2007) 737-754 MR2308962

[3] T Baird, Moduli spaces of vector bundles over a real curve: $\mathbb{Z} / 2-$ Betti numbers, Canad. J. Math. 66 (2014) 961-992 MR3251762

[4] A Borel, Topology of Lie groups and characteristic classes, Bull. Amer. Math. Soc. 61 (1955) 397-432 MR0072426

[5] A M Gleason, Spaces with a compact Lie group of transformations, Proc. Amer. Math. Soc. 1 (1950) 35-43 MR0033830

[6] K Kuribayashi, M Mimura, T Nishimoto, Twisted tensor products related to the cohomology of the classifying spaces of loop groups, Mem. Amer. Math. Soc. 849, Amer. Math. Soc. (2006) MR2203859

[7] S Mohrdieck, R Wendt, Integral conjugacy classes of compact Lie groups, Manuscripta Math. 113 (2004) 531-547 MR2129875

[8] R S Palais, Foundations of global non-linear analysis, W A Benjamin, New York (1968) MR0248880

[9] A Pressley, G Segal, Loop groups, Oxford Math. Monographs, Oxford Univ. Press (1986) MR900587

[10] G Segal, Classifying spaces and spectral sequences, Inst. Hautes Études Sci. Publ. Math. (1968) 105-112 MR0232393

[11] J de Siebenthal, Sur les groupes de Lie compacts non connexes, Comment. Math. Helv. 31 (1956) 41-89 MR0094408

[12] S Stanciu, A geometric approach to D-branes in group manifolds, Fortschr. Phys. 50 (2002) 980-985 MR1942511

[13] R Wendt, Weyl's character formula for non-connected Lie groups and orbital theory for twisted affine Lie algebras, J. Funct. Anal. 180 (2001) 31-65 MR1814422

Department of Mathematics \& Statistics, Memorial University of Newfoundland St. John's NF A1C 5S7, Canada

tbaird@mun.ca

http://www.thomasjohnbaird.com

Received: 8 May 2014 Revised: 25 May 2015 
